\title{
Two Cases of Severe Type 2 Respiratory Failure Associated with Diffuse Idiopathic Skeletal Hyperostosis
}

\author{
Tatsuya Sato, Taro Bannai, Toru Miyake, Keita Murakami, Risa Maekawa, Yasushi Shiio
}

Department of Neurology, Tokyo Teishin Hospital, Tokyo, Japan

Corresponding Author:

Tatsuya Sato, MD

Department of Neurology, Tokyo

Teishin Hospital, 2-14-23 Fujimi,

Chiyoda-ku, Tokyo 102-8798, Japan

E-mail: tatsuyasato0611@gmail.com

ORCID:

https://orcid.org/0000-0003-0208-8010

Received: December 28, 2020

Revised: January 23, 2021

Accepted: February 5, 2021
Diffuse idiopathic skeletal hyperostosis (DISH) is a non-inflammatory process characterized by hyperostosis at tendon insertions and around joint capsules and ossification of the anterior longitudinal ligament of the spine. The flexibility of the spinal column is reduced in DISH and affects the movement of the thorax, leading to restrictive ventilatory function. In this report, we describe the first two cases of severe type 2 (hypercapnic) respiratory failure associated with DISH. Two older men presented with histories of shortness of breath. Radiography of the spine revealed DISH with coexisting ankylosis of the costovertebral joints. The patients' thoracic motion was severely restricted, reducing the mechanism of lung expansion to diaphragm contraction only. Both patients required non-invasive positive-pressure ventilation therapy to cope with their conditions. Our report sheds light on the risk of potentially life-threatening respiratory manifestations of DISH among older adults.

Key Words: Diffuse idiopathic skeletal hyperostosis, Respiratory insufficiency, Hypercapnia, Positive-pressure ventilation

\section{INTRODUCTION}

Diffuse idiopathic skeletal hyperostosis (DISH) is a non-inflammatory process characterized by hyperostosis at tendon insertions and around joint capsules and ossification of the anterior longitudinal ligament of the spine. The prevalence of DISH is approximately $10 \%$ in persons aged $>50$ years. ${ }^{1)}$ The condition is diagnosed based on the following radiological criteria: the presence of $\geq 4$ consecutive fused vertebral bodies with contiguous ligamentous ossification, preservation of the intervertebral disk space, and absence of gross degeneration in the apophyseal and sacroiliac joints. ${ }^{2)}$ Although various established orthopedic problems are reportedly associated with DISH, including vulnerability to trauma and risk of fractures, ${ }^{3-8)}$ few studies have reported DISH as a cause of restrictive respiratory impairment. ${ }^{9-11)}$ Here, we report two cases of DISH that led to type 2 (hypercapnic) respiratory failure by limiting the respiratory movement of the chest wall. To our knowledge, this is the first report describing patients with severe type 2 respiratory failure associated with $\mathrm{DISH}$, which necessitated non-invasive positive-pressure ventilation therapy (NIPPV).

\section{CASE DESCRIPTION}

\section{Case 1}

An 81-year-old man presented to the neurology department with progressive dyspnea on exertion that started 3 years ago. He experienced cholelithiasis 9 years ago that was surgically treated. His preoperative vital capacity as a percent of predicted $(\% \mathrm{VC})$ was decreased to $61 \%$. His medical history included cervical spondylosis treated by posterior decompression at 62 years of age. He had smoked a pack of cigarettes per day for 50 years and had no exposure to dust or asbestos. The results of a bronchodilator response test ruled out asthma.

The patient's oxygen saturation at rest was $95 \%$ and dropped to $85 \%$ while walking. His respiratory sounds were normal on auscultation. Arterial blood gas analysis revealed a $\mathrm{pH}$ of 7.33, partial 
pressure of oxygen $\left(\mathrm{PaO}_{2}\right)$ of $79 \mathrm{mmHg}$, and partial pressure of carbon dioxide $\left(\mathrm{PaCO}_{2}\right)$ of $65 \mathrm{mmHg}$ while breathing ambient air. His bicarbonate $\left(\mathrm{HCO}_{3}^{-}\right)$level was elevated $(30.1 \mathrm{mEq} / \mathrm{L})$, indicating metabolic compensation for respiratory acidosis. His body mass index (BMI) was $14.2 \mathrm{~kg} / \mathrm{m}^{2}$ and his \%VC decreased to $39.1 \%$ in respiratory function tests (Table 1 ). The patient's forced

Table 1. Results of the respiratory function test in Case 1

\begin{tabular}{lcc}
\hline & Volume (L) & $\%$ \\
\hline Respiratory function test & & 39.4 \\
VC & 1.15 & \\
IC & 0.64 & \\
IRV & 0.31 & \\
TV & 0.33 & 40.8 \\
ERV & 0.51 & 105.9 \\
TLC & 5.22 & 103.2 \\
FRC & 4.58 & 184.2 \\
RV & 4.07 & 174.1 \\
RV/TLC (\%) & 78.0 & \\
Spirometry & & 39.0 \\
FVC & 1.14 & 64.4 \\
FEV $_{1}$ & 1.12 & \\
FEV $_{1} \%$ & 98.2 & \\
\hline
\end{tabular}

VC, vital capacity; IC, inspiratory capacity; IRV, inspiratory reserve volume; TV, tidal volume; ERV, expiratory reserve volume; TLC, total lung capacity; FRC, functional residual capacity; RV residual volume; FVC, forced vital capacity; $\mathrm{FEV}_{1}$, forced expiratory volume in one second; $\mathrm{FEV}_{1} \%$, forced expiratory volume in one second as a percent of predicted. expired volume in 1 second as a percent of predicted $\left(\mathrm{FEV}_{1} \%\right)$ remained unchanged. His tidal volume decreased to $300 \mathrm{~mL}$ (normal range, $350-500 \mathrm{~mL}$ ), while his minute volume remained at $7.71 \mathrm{~L} / \mathrm{min}$ (normal range, $5-9 \mathrm{~L} / \mathrm{min}$ ) owing to his increased respiratory rate. The significant increase in the residual volume (RV) compared with the unchanged total lung capacity (TLC) resulted in an elevated RV/TLC. Although the findings of neurological examinations, including manual muscle testing (MMT) and grip strength and electromyography of the extremities, were normal, he could not bend backward owing to reduced spinal mobility (Fig. 1A). Thoracic movement was hardly observable even while taking deep breaths. Radiography of the lumbar spine showed non-marginal syndesmophytes on the anterior and lateral aspects of the vertebral columns with a "flowing candle wax" appearance, while the intervertebral spaces were preserved (Fig. 1B1D). We observed degeneration of the cervical spine with bone spurs; however, we found no lesions causing upper airway obstruction (Fig. 1E). Computed tomography (CT) revealed longitudinal ossification of the anterior longitudinal ligament in the thoracolumbar spine and ankylosis of the costovertebral joints (Fig. 1F, $1 \mathrm{G}$ ) but no abnormalities in the lung fields or pleura. Moreover, we observed no ankylosis in the apophyseal and sacroiliac joints. Therefore, we diagnosed the patient as having DISH associated with restrictive respiratory impairment, which eventually led to type 2 respiratory failure. Introduction of NIPPV that was adjusted to the patient's relatively high respiratory rate relieved his symp-
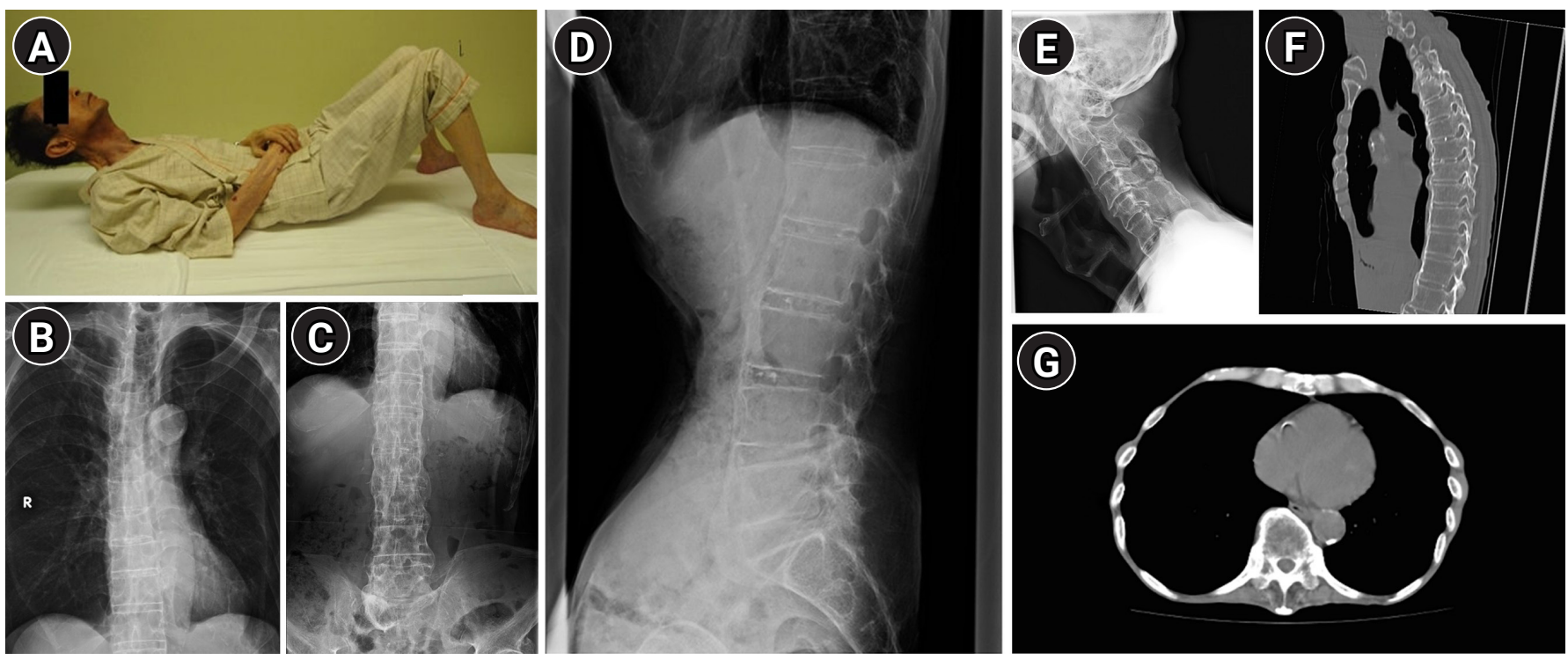

Fig. 1. (A) Because of reduced spinal mobility, the patient could not touch the mattress with the back of his head while lying down. (B-D) Radiography of the spine showing osteophytes on the anterior and lateral aspects of the vertebral columns with preserved intervertebral disc spaces. (E) Radiography of the cervical spine showing degeneration of the vertebral bodies with bone spurs. No upper airway obstruction owing to diffuse idiopathic skeletal hyperostosis lesions are observed. (F, G) Chest computed tomography showing longitudinal ossification of the anterior longitudinal ligament and hyperostosis of the thoracic costovertebral joints. 
toms. One year following his discharge, his findings remain stable.

\section{Case 2}

An 89-year-old man with a chief complaint of shortness of breath presented to our hospital. His symptoms had begun 1 week before and had progressively worsened. He had a medical history of hypertension and cervical spinal stenosis that had been treated surgically. His social history was positive for smoking tobacco (40 packyears), although he had quit smoking 30 years ago. He had no history of exposure to dust or asbestos.

On arrival, the patient's blood pressure was $153 / 84 \mathrm{mmHg}$ and his pulse was $86 \mathrm{bpm}$. His respiratory rate was $26 / \mathrm{min}$, and his oxygen saturation while breathing ambient air was $70 \%$, which increased to $90 \%$ with a $3-\mathrm{L}$ nasal cannula. His BMI was $21.3 \mathrm{~kg} / \mathrm{m}^{2}$. We observed distention of his jugular veins and edema in his lower extremities. Arterial blood gas analysis revealed a $\mathrm{pH}$ of 7.24, $\mathrm{PaO}_{2}$ of $45 \mathrm{mmHg}$, and $\mathrm{PaCO}_{2}$ of $107 \mathrm{mmHg}$ with a 0.75 - $\mathrm{L}$ nasal cannula. $\mathrm{His} \mathrm{HCO}_{3}{ }^{-}$level was elevated $(45.4 \mathrm{mEq} / \mathrm{L})$. Blood test results revealed markedly increased brain natriuretic peptide levels (920 pg/mL) (normal < 18.4 pg/ mL). Electrocardiography showed a normal sinus rhythm. Echocardiography showed a moderately elevated tricuspid regurgitation velocity, indicating right heart strain. Chest radiography showed cardiac enlargement accompanied by bilateral pleural effusion. Chest CT showed no abnormalities in the lung fields other than pulmonary arterial dilata-
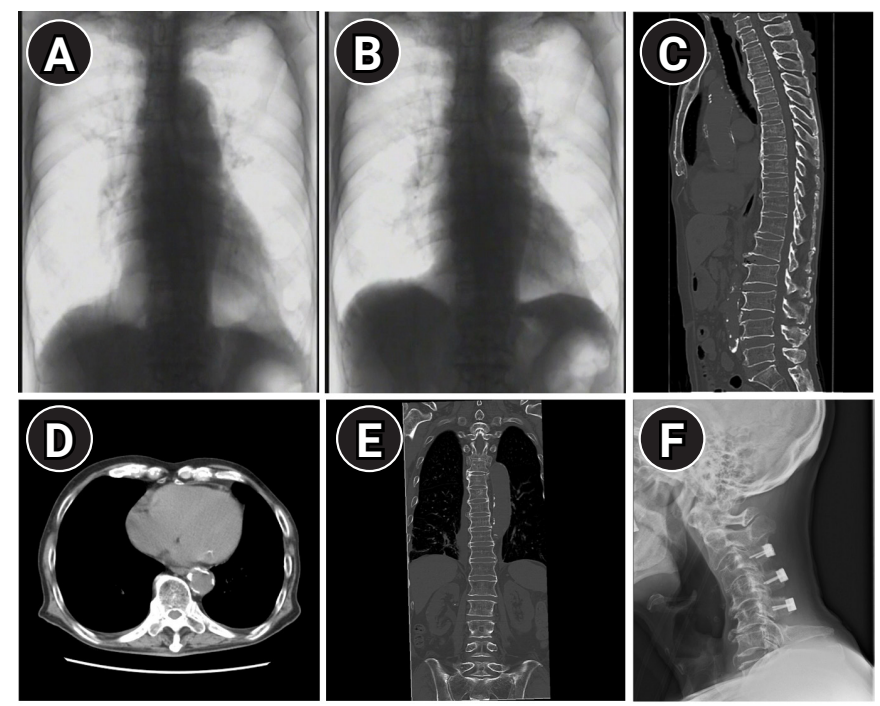

Fig. 2. Movement of the thoracic cage between inspiration (A) and expiration (B) was not observed, other than diaphragmatic contraction on fluoroscopy (still images). Trunk computed tomography showing longitudinal ossification of the anterior longitudinal ligament in the thoracic spines (C) and ankylosis of the costovertebral joints (D). (E) The sacroiliac joints are intact. (F) Radiography of the cervical spine showing no lesions causing upper airway obstruction on the anterior aspect of the vertebrae. tion and bilateral pleural effusion.

The patient was diagnosed with congestive heart failure and started treatment with NIPPV and diuretics. After 10 days in the intensive care unit, his symptoms had resolved and arterial blood gas analysis showed a $\mathrm{pH}$ of $7.39, \mathrm{PaO}_{2}$ of $77 \mathrm{mmHg}$, and $\mathrm{PaCO}_{2}$ of $53 \mathrm{mmHg}$ with a $0.5-\mathrm{L}$ nasal cannula. While his $\mathrm{HCO}_{3}{ }^{-}$level decreased to $29.6 \mathrm{mEq} / \mathrm{L}$, it still indicated metabolic compensation for respiratory acidosis. His weight dropped to his regular weight of $48 \mathrm{~kg}\left(\mathrm{BMI} 18.3 \mathrm{~kg} / \mathrm{m}^{2}\right)$. The respiratory function test conducted following recovery from the initial respiratory failure revealed low \%VC and $\mathrm{FEV}_{1} \%$ (Table 2). Examination of his nervous system, including MMT and grip strength, showed no abnormalities. We detected no abnormal lung sounds, such as stridor or wheeze, on auscultation. Needle electromyography showed no significant changes. Chest fluoroscopy showed significantly reduced movements of the thoracic cage, with deep breathing and diaphragmatic contraction seemingly the only mechanisms contributing to his respiratory motion (Fig. 2A, 2B). The difference in chest measurements at the fourth intercostal space between inspiration and expiration was only $0.5 \mathrm{~cm}$ (lower limit of normal, $2.5 \mathrm{~cm}$ ), reflecting the restricted thoracic motion. Trunk CT revealed longitudinal ossification of the anterior longitudinal ligament in the thoracic spine and ankylosis of the costovertebral joints without signs of degeneration in the sacroiliac joints (Fig. 2C-2E). Based on these findings, the patient's underlying restrictive respiratory impairment was attributed to DISH. We observed no lesion causing upper airway obstruction on the anterior aspect of the cervical

Table 2. Results of the respiratory function test in Case 2

\begin{tabular}{lcc}
\hline & Volume (L) & $\%$ \\
\hline Respiratory function test & & \\
VC & 1.78 & 59.3 \\
IC & 0.92 & \\
IRV & 0.33 & \\
TV & 0.59 & \\
ERV & 0.86 & 66.2 \\
TLC & 4.44 & 84.6 \\
FRC & 3.52 & 75.2 \\
RV & 2.66 & 99.3 \\
RV/TLC (\%) & 59.9 & 117.1 \\
Spirometry & & \\
FVC & 1.80 & 60.0 \\
FEV $_{1}$ & 0.87 & 45.5 \\
FEV $_{1} \%$ & 48.3 & \\
\hline
\end{tabular}

VC, vital capacity; IC, inspiratory capacity; IRV, inspiratory reserve volume; TV, tidal volume; ERV, expiratory reserve volume; TLC, total lung capacity; FRC, functional residual capacity; RV residual volume; FVC, forced vital capacity; $\mathrm{FEV}_{1}$, forced expiratory volume in one second; $\mathrm{FEV}_{1} \%$, forced expiratory volume in one second as a percent of predicted. 
spine (Fig. 2F). The patient was discharged under NIPPV therapy. Six months after discharge, he remains independent in his activities of daily living and lacks signs of heart failure, although blood gas analysis shows persistent hypercapnia with metabolic compensation.

All author declare that written informed consent was obtained from the patient for publication of this case report and accompanying image.

\section{DISCUSSION}

The flexibility of the spinal column is reduced in patients with DISH owing to the ossification of the anterior longitudinal ligament. The coexistence of ankylosis of the costovertebral joints with ossification further restricts thoracic motion, reducing the mechanism of lung expansion to diaphragmatic contraction only, ${ }^{9,10)}$ In ankylosing spondylitis (AS), which shares the same features of spinal ankylosis with DISH, ankylosis of the joints reduces the compliance of the thorax, fixing the thorax in the extended position. ${ }^{12,13)}$ These changes lead to decreased vital capacity and relatively increased RV with preserved TLC. Decreased alveolar ventilation volume leads to increased $\mathrm{PaCO}_{2}$. These features were observed on spirometry in both of our cases. In Case 1, the preserved minute volume during respiratory function tests with a compensatory increase in respiratory rate indicated no apparent loss of strength in the respiratory muscles themselves. However, there was a limit to the increase in respiratory rate during continuous movement. The patient could not compensate for increased oxygen demand owing to persistent tachypnea; thus, his oxygen saturation $\left(\mathrm{SpO}_{2}\right)$ decreased while walking and other daily activities. We also observed increased RV/TLC with relatively preserved TLC along with severe restrictive respiratory impairment in Case 2. The ossification of the anterior longitudinal ligament observed in the two patients was not as bulky as previously reported in patients with DISH; ${ }^{14)}$ however, there were no signs of sacroiliitis, which is the hallmark sign of AS. Additionally, both patients denied experiencing back pain when they were young. Considering these facts and their age, we concluded that DISH rather than AS was the underlying condition for restrictive respiratory impairment. Severe upper respiratory tract obstruction caused by cervical osteophytes is a mechanism of respiratory failure related to DISH. ${ }^{15)}$ However, neither patient had lesions obstructing their upper respiratory tracts. The present cases indicate that restrictive ventilatory function caused by thoracic lesions may lead to severe respiratory failure in patients with DISH. In line with previous reports, ${ }^{9,10)}$ we assume that ankylosis of the costovertebral joints contributes to severe respiratory dysfunction. The patients did not take medications that may have caused drug-induced hypercapnia, such as tranquilizers or painkillers.

The pattern of spirometry was similar to that observed in patients with amyotrophic lateral sclerosis (ALS). ${ }^{16)}$ The two patients described here were evaluated in our neurology department for suspected motor neuron disease with respiratory onset. However, needle electromyography did not show a progressive denervation process, which is the hallmark of ALS, ${ }^{17}$ in either patient. Although neuromuscular diseases are major extrinsic factors of restrictive spirometric patterns, physicians should also pay attention to abnormalities in vertebral bodies, especially after ruling out common restrictive causes. In Case 2, reduced $\mathrm{FEV}_{1} \%$ was also observed, although no signs of emphysema were noted on chest CT. Based on the patients' smoking history and right heart strain indicated by echocardiography, he might have had non-emphysematous chronic obstructive pulmonary disease. However, the use of bronchodilators and inhaled corticosteroids did not relieve his respiratory symptoms. Moreover, signs of asthma, such as persistent coughing and wheezing sounds, were not observed. Based on a study reporting the association of DISH with reduced $\mathrm{FEV}_{1}{ }^{18)}$ we speculate that DISH affected the patient's expiratory ability and vital capacity.

To our knowledge, these are the first two reported cases of DISH associated with severe type 2 respiratory failure, although the prevalence rate of thoracic DISH is high among older adults. Along with skeletal problems limiting thoracic motion, the low $\mathrm{BMI}$ in this population might be related to adverse outcomes because physical frailty is a known aggravator of respiratory diseases. ${ }^{19)}$ However, despite the severity of their respiratory failure, the muscle strength in the extremities, including grip strength, in these patients remained normal on MMT, and they did not report unintentional weight loss at the time of diagnosis. Thus, we concluded that DISH restricting thoracic motion rather than physical frailty was the leading cause of their restrictive ventilatory function. NIPPV allowed the patients to perform their activities of daily living.

Although DISH is a relatively common condition among older adults, its effect on respiratory function is underestimated. Herein, we illustrated the importance of focusing on thoracic movement and explicitly evaluating DISH when physicians encounter cases with restrictive respiratory impairment of unknown etiology. Restrictive respiratory impairment caused by the disease can follow a severe life-threatening clinical course, as described in the present report. 


\section{ACKNOWLEDGMENTS}

\section{CONFLICT OF INTEREST}

The researchers claim no conflicts of interest.

\section{FUNDING}

None.

\section{AUTHOR CONTRIBUTION}

Patient care, TS, TB, TM, KM, RM, YS; Writing-original draft, TS; Writing-review \& editing, TB, RM, YS.

\section{REFERENCES}

1. Mader R, Verlaan JJ, Eshed I, Bruges-Armas J, Puttini PS, Atzeni F, et al. Diffuse idiopathic skeletal hyperostosis (DISH): where we are now and where to go next. RMD Open 2017;3:e000472.

2. Resnick D, Niwayama G. Radiographic and pathologic features of spinal involvement in diffuse idiopathic skeletal hyperostosis (DISH). Radiology 1976;119:559-68.

3. Shin BS, Seo MW, Kim YH. A case quadriplegia due to minor head trauma associated with ossification of the posterior longitudinal ligament of the cervical spine. J Korean Geriatr Soc 2002;6:74-8.

4. Westerveld LA, Verlaan JJ, Oner FC. Spinal fractures in patients with ankylosing spinal disorders: a systematic review of the literature on treatment, neurological status and complications. Eur Spine J 2009; 18:145-56.

5. Caron T, Bransford R, Nguyen Q, Agel J, Chapman J, Bellabarba C. Spine fractures in patients with ankylosing spinal disorders. Spine (Phila Pa 1976) 2010;35:E458-64.

6. Liu P, Yao Y, Liu MY, Fan WL, Chao R, Wang ZG, et al. Spinal trauma in mainland China from 2001 to 2007: an epidemiological study based on a nationwide database. Spine (Phila Pa 1976) 2012;37:1310-5.

7. Mazieres B. Diffuse idiopathic skeletal hyperostosis (Forestier-Rotes-Querol disease): what's new? Joint Bone Spine 2013; 80:466-70.

8. Tripathi M, Rajmohan D, Quirk C, Beckett B, Choi D, RichGarg N, et al. diffuse idiopathic skeletal hyperostosis, associated morbidity, and healthcare utilization: a university hospital experience. J Clin Rheumatol 2020;26:104-8.

9. Yasuda T, Suzuki K, Yahara Y, Watanabe K, Kawaguchi Y. Relation between costovertebral joint change and respiratory function in patients with diffuse idiopathic skeletal hyperostosis (DISH). Spine Spinal Cord 2019;32:661-6. https://doi.org/10. $11477 / \mathrm{mf} .5002201172$.

10. Yoshida M, Kibe A, Aizawa H, Matsumoto K, Inoue H, Koto H, et al. Diffuse idiopathic skeletal hyperostosis with fibrobullous change in upper lung lobes and dyspnea due to limitation of thoracic cage. Nihon Kokyuki Gakkai Zasshi 1999;37:823-8.

11. Oudkerk SF, Mohamed Hoesein FA, Oner FC, Verlaan JJ, de Jong PA, Kuperus JS, et al. Diffuse idiopathic skeletal hyperostosis in smokers and restrictive spirometry pattern: an analysis of the COPDGene Cohort. J Rheumatol 2020;47:531-8.

12. Luthra HS. Extra-articular manifestations of ankylosing spondylitis. Mayo Clin Pro 1977;52:655-6.

13. Feltelius N, Hedenstrom H, Hillerdal G, Hallgren R. Pulmonary involvement in ankylosing spondylitis. Ann Rheum Dis 1986; 45:736-40.

14. Mader R, Baraliakos X, Eshed I, Novofastovski I, Bieber A, Verlaan JJ, et al. Imaging of diffuse idiopathic skeletal hyperostosis (DISH). RMD Open 2020;6:e001151.

15. Verlaan JJ, Boswijk PF, de Ru JA, Dhert WJ, Oner FC. Diffuse idiopathic skeletal hyperostosis of the cervical spine: an underestimated cause of dysphagia and airway obstruction. Spine J 2011; 11:1058-67.

16. Chandrasoma B, Balfe D, Naik T, Elsayegh A, Lewis M, Mosenifar Z. Pulmonary function in patients with amyotrophic lateral sclerosis at disease onset. Monaldi Arch Chest Dis 2012;77:12933.

17. de Carvalho M, Dengler R, Eisen A, England JD, Kaji R, Kimura J, et al. Electrodiagnostic criteria for diagnosis of ALS. Clin Neurophysiol 2008; 119:497-503.

18. Oudkerk SF, Buckens CF, Mali WP, De Koning HJ, Oner FC, Vliegenthart R, et al. Diffuse Idiopathic Skeletal Hyperostosis Is Associated with Lower Lung Volumes in Current and Former Smokers. Am J Respir Crit Care Med 2016;194:241-2.

19. Bone AE, Hepgul N, Kon S, Maddocks M. Sarcopenia and frailty in chronic respiratory disease. Chron Respir Dis 2017; 14:85-99. 